\title{
Orchids as Potential Sources of Anticancer Agents: Our Experience
}

\author{
Bijaya Pant ${ }^{1,2}$, Mukti Ram Paudel ${ }^{1}$, Pusp Raj Joshi ${ }^{1,2}$ \\ ${ }^{1}$ Central Department of Botany, Tribhuvan University, Kirtipur, Kathmandu, Nepal \\ ${ }^{2}$ Annapurna Research Center, Maitighar, Kathmandu, Nepal
}

\section{CORRESPONDENCE}

Prof.Bijaya Pant

Central Department of Botany,

Tribhuvan University, Kirtipur,

Kathmandu, Nepal

Email: b.pant@cdbtu.edu.np

\section{ARTICLE INFO}

Article History

Submitted: 01 October, 2020

Accepted: 29 December, 2020

Published: 8 February,2021

Source of support: None

Conflict of Interest: None

Copyright : (CThe Author(S) 2020

This is an open access article under the Creative Common Attribution license CC-BY 4.0

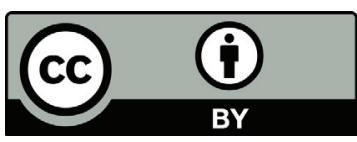

\begin{abstract}
Cancer is the second leading cause of death globally and the cancer burden continues to grow, exerting tremendous physical, emotional and financial strain on individuals, families, communities and health systems. Many health systems in low- and middle-income countries are ill-prepared to manage this burden, and around the world large numbers of cancer patients do not have access to timely quality diagnosis or treatment. Natural products, especially originated from plants, have been applied as remedies to treat various diseases, including cancer, for thousands of years. Several anticancer agents derived from plants, such as paclitaxel (taxol), vinblastine, vincristine, camptothecin derivatives, podophyllotoxins, are in clinical use and a number of other promising agents are in preclinical or clinical development. A growing number of bioactive compounds with cytostatic and cytotoxic activities to treat cancer have been isolated from several orchid species. The Annapurna Research Center in collaboration with other academic institutions is engaged in identifying and exploring anticancer compounds in orchids of Nepal. The center has identified ten different orchid species which contain high phenol and flavonoid contents evidently showing anticancer activities in our in vitro assays against different cancer cell lines viz cervical cancer, lung cancer and brain tumor cell lines. These findings demonstrate that orchids which have been used in in various formulations in traditional medicine since ancient times qualify as potential source for novel drug candidates or starting points for further lead optimization towards clinical candidates for the most urgently needed treatment of aggressive types of cancer. This review paper highlights that in all likelihood; our contributions in orchid research here in Nepal could bring great relief to patients with cancer globally.
\end{abstract}

Key words: Cancer; Orchids; Anticancer compounds; Natural products

\section{INTRODUCTION}

Cancer is a group of over 100 disease types leading to abnormal invading cell growth, which spreads to other parts in the body, referred to as metastasizing. Metastases are a major cause of death from cancer. Globally, cancer is one of the leading causes of death. Worldwide, one in five men and one in six women develop cancer during their lifetimes. The five-year-prevalence figure is estimated to be 43.8 million cases globally and is expected to rise by additional 22 million annually within the next two decades. ${ }^{1}$ The International Agency for Research on Cancer (IARC) released on 14th December the updated Globocan 2020 with new estimates on the global cancer burden, indicating that it has risen to 19.3 million cases and 10 million cancer deaths in $2020 .{ }^{2}$ Treatment of cancer currently includes the surgical removal of cancerous tissue, radiotherapy, chemotherapy and a combination of chemotherapy and targeted therapy. Many health systems in low- and middle-income countries are least prepared to manage this burden, and large numbers of cancer patients globally do not have access to timely quality diagnosis and treatment. The WHO reported that $30 \%$ of the deaths from cancer are preventable if people would not consume tobacco products and if they would eat a healthy diet, exercise regularly, limit the use of alcohol and protect themselves against cancercausing infections. ${ }^{1}$ The utilization of anti-cancer drugs in chemotherapy, while often more beneficial when used in conjugation with radiation therapy or surgery, is considered the first line of treatment. Because cytotoxic drugs are the mainstay of chemotherapy, it is important to discover novel cytotoxic or cytostatic agents with sufficient activity, novel mechanisms-of-action and minimal issues of toxicity and amenable to targeted therapies. ${ }^{3,4,5}$

Natural products, especially derived from plants, have been used to treat various diseases for thousands of years. The world market for medicinal plants and their products is increasing with rapidly escalating prices. The demand 
for medicinal plants intended for use by traditional Ayurveda and modern pharmaceutical companies as source for novel drug candidates is on the rise. Therefore, the trade of medicinal plants becomes an important source of revenue to the Nepal government and supports substantially the livelihoods of communities, particular in rural settings. Medicinal plants have enormous therapeutic potential and may have few side effects. ${ }^{6}$ The search for anticancer agents from plant sources started back in the 1950s, when cytotoxic vinca alkaloids (vinblastine and vincristine) and podophyllotoxins (etoposide and teniposide) were isolated. ${ }^{7}$ Furthermore, several anticancer agents derived from plants, including taxol (Taxus brevifolia), vinblastineand vincristine(Catharanthus roseus), camptothecin derivatives (Camptotheca acuminata), podophyllotoxins (Podophyllum peltatum) are in clinical use, and followed by a number of promising agents in preclinical or clinical development. Examples are flavopiridol, roscovitine, combretastatin A-4, betulinic acid and silvestrol. Since synthetic anticancer drugs are expensive and not easy to access for everybody in Nepal. There is a need to search for novel anticancer compounds in plants, particular herbs, which are rich in compounds with high polyphenol and flavonoid contents. Compounds rich in polyphenol and flavonoids exhibit high antioxidant activities, which property plays an important role for the treatment of cancers. ${ }^{8}$
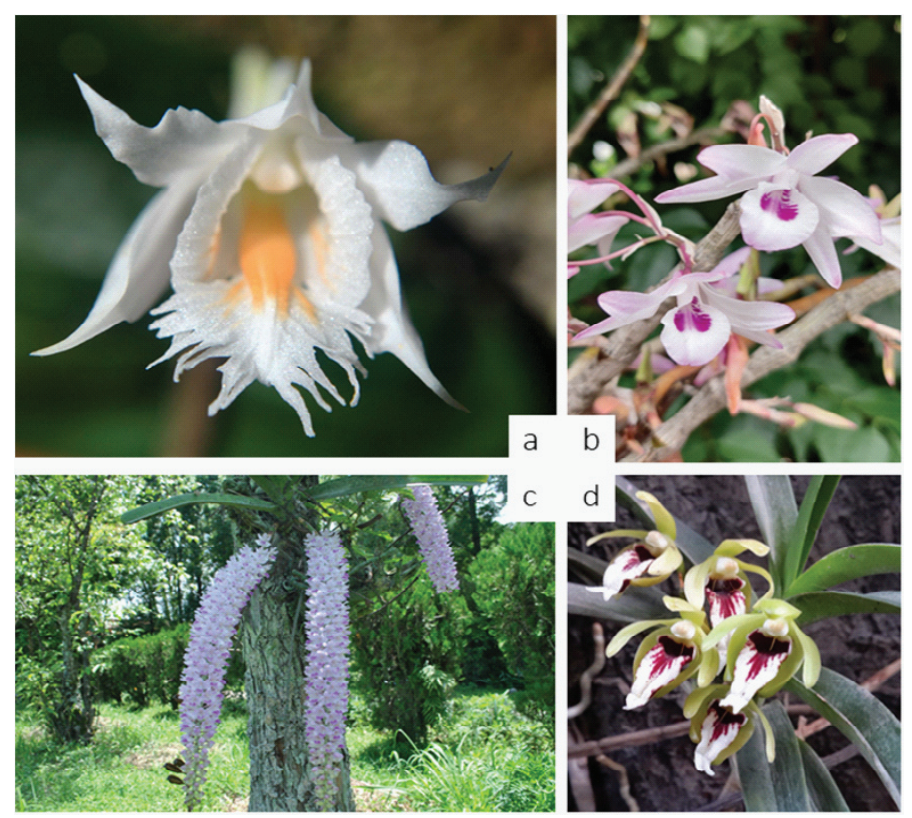

Figure 1: Some orchid species of anticancer sources; Dendrobium longicornu (a), D. transparens (b), Rhyncostylish retusa (c) and Vanda cristata (d).

For this reason, Annapurna Research Center, in collaboration with various other academic institutions, is engaged in identifying and exploring anticancer compounds isolated from orchids, which are used in traditional medicine in various formulations. The center examines in vitro dose-dependent effects of orchid extracts with a high phenol and flavonoid content against cancercell lines, namely for glioblastoma, cervical and lung cancer. It is determined whether or not the compounds extracted arrests the proliferation of cancerous cells. In result ten different orchid species were identified where their extracts demonstrated strong antiproliferative activities in the assays. Some orchids of Nepal (Fig.1) have already been investigated for their anticancer activity in different cancer-cell lines. ${ }^{8-11}$ Once there is a suggestion that antioxidants and anticarcinogens do indeed exist in a particular plant, it has been investigated further, using crude extracts from various plant parts as well as in vitro culture-raised tissue. Moreover, the center is conserving the germplasm of species with anticancer potential using in vitro culture technologies. Some of the species are amenable to mass propagation. Such highly interesting process of cultivation has been exercised in a partnering mode with different stakeholders of society.

\section{Anticancer agents from orchids}

A large number of bioactive compounds with anticancer and anti-tumorous activities have been isolated from several orchid species (Table 1). The methanolic extract obtained from Anoectochilus formosanus have shown to induce apoptosis of MCF-7 cells. Antitumor activity of A. formosanus may be associated with its potent immune stimulating effect. ${ }^{12}$ A phenanthrene derivative 3,7dihydroxy- 2,4,6trimethoxyphenanthrene was isolated from the all plant of Bulbophyllum odoratissimum. The compound displayed cytotoxicity against the growth of human leukemia cell lines K562 and HL-60, human lung adenocarcinoma A549, human hepatoma BEL7402 and human stomach cancer cell lines SGC-7901 with IC50 values of $14.23,10.02,3.42,15.36$ and 1.13 $\mathrm{mg} / \mathrm{ml}$ respectively. ${ }^{13}$ A number of compounds such as 7,8-dihydro-4-hydroxy 12,13-methylenedioxy-11methoxyldibenz[bf]oxepin, cumulating, densiflorol $A$ and plicatol B isolated from Bulbophyllum kwangtungense have shown anti-tumor activities against Hela and K562 human tumor cell lines (Wang et al., 2006). Silbenoids, 3,3'-dihydroxy-2',6'-bis(p-hydroxybenzyl)-5methoxybibenzyl and 3',5-dihydroxy-2-(p-hydroxybenzyl)3-methoxybibenzyl isolated from the tubers of methanol extract of Bletilla striata showed inhibitory effect of tubulin polymerization at IC50 $10 \mu \mathrm{M}$. On the other hand, phenanthrene and dihydrophenanthrene with a benzyl moiety (1-(p-hydroxybenzyl)-4,8-imethoxyphenanthrene2,7-diol and 2,7-dihydroxy-1,3-bis(phydr-oxybenzyl)4-methoxy-9,10-dihydrophenanthrene), and dimeric 
phenanthrenes blestriarenes $\mathrm{B}$ and $\mathrm{C}$ and blestrianol A were found to be three times less potent (IC50: 30 $\mu \mathrm{M})$ than bisbenzyls indicating that the restricted diaryl ring system of phenanthrene is unfavorable for tubulin binding. Furthermore, bisbenzyl 3,3'-dihydroxy-2',6'bis(p-hydroxybenzyl)-5-methoxybibenzyl potentiated the cytotoxicity of SN-38 in BCRP-transduced $\mathrm{K} 562$ (K562/BCRP) cells. ${ }^{14}$ Studies were also carried out on microspheres of tubers of $B$. striata on angiogenesis. It produced a change of tumor microcirculation after transcatheter arterial chemoembolization: first pass perfusion MR imaging and Chinese ink casting in a rabbit model. With Bletilla microspheres may enhance its anti-tumor effect by inhibiting the angiogenesis (Zhao et al., 2004). The same observations have been made in other experiment when tested $B$. striata colloid on angiogenesis. Inhibits angiogenesis, through binding inhibition of vascular endothelial growth factor to its receptor. ${ }^{15}$

The tuber of Cremastra appendiculata yield cirrohopetalanthrin and $2,7,2^{\prime}, 7^{\prime}, 2^{\prime \prime}$-pentahydroxy$4,4^{\prime} 4^{\prime \prime} 7^{\prime \prime}$-tetramethoxy-1,8,1',1' -triphenanthrene which were found to have moderate cytotoxicity against human colon cancer, human stomach cancer, human hepatoma, human breast cancer, human lung adenocarcinoma and human ovarian cancer cell lines. ${ }^{16}$ The homoisoflavanone 5,7-dihydroxy-3-(3-hydroxy-4methoxybenzyl)-6 methoxychroman isolated from $C$. appendiculata was found to be a potent inhibitor of angiogenesis. ${ }^{17}$ Cephalinone F isolated from Cephalanceropsis gracilis, a native orchid of Taiwan, has significant cytotoxicity against human breast carcinoma (MCF-7), lung carcinoma ( $\mathrm{NCl}-\mathrm{H} 460)$, and central nervous system carcinoma (SF268) cell lines. Cirrhopetalanthrin isolated from tuber of Cremastra appendiculata showed non-selective moderate cytotoxicity with IC50 values of 8.4-13.3 $\mu \mathrm{M}$ against human colon cancer (HCT-8), human hepatoma (Bel7402), human stomach cancer (BGC-823), human lung adenocarcinoma (A549), human breast cancer (MCF-7) and human ovarian cancer (A2780) cell lines. ${ }^{17}$ A study to understand the molecular basis underlying the antitumor effects of $C$. appendiculata was performed by 5,7-dihydroxy-3-(3hydroxy-4-methoxybenzyl)-6-methoxychroman isolated from the bulb. Similar observations were obtained in a study with the ethanol extract of the tuber of $C$. appendiculata was subjected to column chromatography to yield 2,7,2',7',2'-pentahydroxy-4,4',4', $7^{\prime \prime}$-tetramethoxy$1,8,1^{\prime}, 1^{\prime \prime}$ - triphenanthrene and cirrhopetalanthin. Both compounds were evaluated against six human cancer cells; human lung adenocarcinoma (A549), human ovarian cancer (A2780), hepatoma (Bel7402), human stomach cancer (BGC-283), human colon cancer (HTC-8), and human breast cancer (MCF-7) cell lines. Both compounds were selectively active against the human cancer cell lines used. ${ }^{16}$

Denbinobin and 4,7-dihydroxy-2-methoxy-9,10dihydrophenanthrene from Dendrobium nobile showed cytotoxicity against human lung carcinoma, human ovary adenocarcinoma and human promyelocytic leukemia cell lines. ${ }^{17}$ Erianin obtained from the stem of Dendrobium chrysanthum was found to be a potent inhibitor of proliferation of HL-60 cells and the inhibition might be due to erianin-induced apoptosis and altered expression of bcl-2 and bax genes in HL-60 cells. ${ }^{18}$ In another study, erianin leads to extensive tumor necrosis, growth delay and rapid vascular shutdown in hepatoma Bel7402 and melanoma A375. ${ }^{19}$ Dendrochrysanene isolated from stems of $D$. chrysanthum was found to suppress the mRNA level of TNF-alpha, IL8, IL10 and iNOS in murine peritoneal macrophages. ${ }^{20}$ Two pimaranediterpenoids, lonchophylloids A and B were isolated from the stems of Ephemerantha lonchophylla expressed the multidrug resistance phenotype to the toxicity of the anticancer isolated from the stems of Ephemerantha lonchophylla expressed the multidrug resistance phenotype to the toxicity of the anticancer drug doxorubicin. Subsequently, it has been reported that denbinobin also isolated from this plant exhibit anti-tumor and anti-inflammatory activity. Denbinobin displays anticancer effects in K562 cells through the increase of levels of tubulin polymerization and deregulation of BcrAbl signaling (Huang et al., 2005). Methanolic extract of Gastrodia elata prevents serum deprived apoptosis through activation of serine/threonine kinase dependent pathway and suppression of JNK activity. ${ }^{21}$ whereas the ethanolic extract from the rhizomes have shown potent anti-tumor activity in vitro in a dose dependent manner. ${ }^{22}$ According to Huang et al. (2007), bis-(4-Hydroxybenzyl) sulfide and N6-(4-hydroxybenzyl) adenine riboside were isolated from methanol extract of rhizomes of G. elata potently prevented PC12 cell apoptosis on ischemic/hypoxic model to screen neuroprotective in concentration dependent manners with EC50 values of $7.20 \mu \mathrm{M}$ and 3.7 x 10-8 M, respectively. (2S)5,2',6'-trihydroxy-6-lavandulyl$4^{\prime \prime}$-( $\gamma, \gamma$ dimethylallyl)-2", 2"-dimethylpyrano-[5",6", 7,8$]$ flavanone, a dihydroflavanoid isolated from Spiranthes australis inhibits human cancer cells growth including A498, A549, BEL-7402,SGC-7901,MCF-7, HT-29 and K562 cell lines. ${ }^{23}$ 
Table 1: Anticancer effect of extracts/compounds of different orchid species on different cancer cell lines.

S.N. Orchid Species

Extract/Active Compound

Biological Target

References

1

Agrostophyllum brevi- Callosinin

pes

Majumder et al. $2001,2003^{24,25}$

Anoectochilus

Methanolic extract

MCF-7 cell lines

Tseng et al., $2006^{26}$ formosanus

3 Bletilla formosana

4-Methoxy-9,10-dihydrophenanthrene-1,2,7triol

Lin et al., $2005^{27}$

1-(4-Hydroxybenzyl)-4,7-dimethoxy-9,10-

dihydrophenanthrene-2-ol

1,3,6-tri(4-Hydroxybenzyl)-4-methoxydihydrophenanthrene-2,7-diol

\begin{tabular}{|c|c|c|c|c|}
\hline 4 & B. striata & $\begin{array}{l}\text { 3,3'-Dihydroxy-2',6'-bis(p-hydroxybenzyl)-5- } \\
\text { methoxybibenzyl }\end{array}$ & K562 cell line & Morita et al., $2005^{14}$ \\
\hline \multirow{9}{*}{5} & \multirow[b]{7}{*}{$\begin{array}{l}\text { Bulbophyllum } \\
\text { kwangtungense }\end{array}$} & $\begin{array}{l}\text { 3',5-Dihydroxy-2-(p-hydroxybenzyl)-3-Meth- } \\
\text { oxybibenzyl }\end{array}$ & \multirow[b]{7}{*}{ Hela and K562 cell lines } & \multirow[b]{7}{*}{ Wu et al., $2006^{28}$} \\
\hline & & $\begin{array}{l}\text { 1-(p-Hydroxybenzyl)-4,8-dimethoxyphenan- } \\
\text { threne-2,7-iol }\end{array}$ & & \\
\hline & & $\begin{array}{l}\text { 2,7-Dihydroxy-1,3-bis(p-hydroxybenzyl)-4- } \\
\text { methoxy-9,10-dihydrophenanthrene }\end{array}$ & & \\
\hline & & Blestrianol A & & \\
\hline & & Blestriarene B & & \\
\hline & & Blestriarene $\mathrm{C}$ & & \\
\hline & & Plicatol B & & \\
\hline & & Cumulatin & & \\
\hline & & Densiflorol A & & \\
\hline 6 & B. odoratissimum & 3,7-Dihydroxy-2,4,6-trimethoxyphenanthrene & $\begin{array}{l}\text { K562, HL-60, A549,BEL- } \\
7402 \text { and SGC-7901 cell lines }\end{array}$ & Chen et al., $2007^{13}$ \\
\hline 7 & Coelogyne cristata & Coeloginanthridin & & Majumder et al., $2001^{24}$ \\
\hline
\end{tabular}

\begin{tabular}{|c|c|c|c|c|}
\hline & \multirow{2}{*}{\multicolumn{3}{|c|}{ Coeloginanthrin }} \\
\hline & & & & \\
\hline 8 & C. flaccida & Callosinin & & $\begin{array}{l}\text { Majumder et al., } \\
2001,2003^{24,25}\end{array}$ \\
\hline 9 & C. stricta & Methanol extract & HeLa cell line & Thapa et al., $2020^{10}$ \\
\hline \multirow[t]{2}{*}{10} & \multirow{2}{*}{$\begin{array}{l}\text { Cremastra } \\
\text { appendiculata }\end{array}$} & Cirrhopetalanthin & \multirow{2}{*}{$\begin{array}{l}\text { HCT-8, Bel7402, BGC-823, } \\
\text { A549, MCF- } 7 \text { and A2780 cell } \\
\text { lines }\end{array}$} & Xue et al., $2006^{16}$ \\
\hline & & $\begin{array}{l}\text { 2,7,2',7',2'-Pentahydroxy-4,4',4",7'"-tetram- } \\
\text { ethoxy-1,8,1',1'”-triphenanthrene }\end{array}$ & & \\
\hline 11 & Dendrobium amoenum & Methanol extract & HeLa and U-251 cell lines & Paudel\& Pant, $2017^{29}$ \\
\hline \multirow[t]{4}{*}{12} & \multirow[t]{4}{*}{ D. brymerianum } & Moscatilin & \multirow[t]{4}{*}{ H460 cell line } & \multirow{4}{*}{$\begin{array}{l}\text { Klongkumnuankarn et al., } \\
2015^{30}\end{array}$} \\
\hline & & Gigantol & & \\
\hline & & Lusianthrindin & & \\
\hline & & Dendroflorin & & \\
\hline \multirow[t]{2}{*}{13} & \multirow[t]{2}{*}{ D. catenatum } & Protein extract & \multirow{2}{*}{$\begin{array}{l}\text { HepG2, SGC-7901, MCF-7 } \\
\text { cell lines }\end{array}$} & \multirow[t]{2}{*}{ Zheng et al., $2015^{31}$} \\
\hline & & Peptides & & \\
\hline \multirow[t]{2}{*}{14} & \multirow[t]{2}{*}{ D. crepidatum } & Methanol extract & \multirow{2}{*}{$\begin{array}{l}\text { HeLa, U-251 cell lines and } \\
\text { T-cell lymphoma }\end{array}$} & \multirow{2}{*}{$\begin{array}{l}\text { Paudel et al., 201932; } \\
\text { Prasad \& Koch, } 2016^{33}\end{array}$} \\
\hline & & Ethanol extract & & \\
\hline \multirow[t]{2}{*}{15} & \multirow[t]{2}{*}{ D. chrysanthum } & Ethanol extract & \multirow{2}{*}{$\begin{array}{l}\text { T-cell lymphoma and } \mathrm{FaDu} \\
\text { cell line }\end{array}$} & \multirow{2}{*}{$\begin{array}{l}\text { Nam et al., 201934; Prasad } \\
\& \text { Koch, } 2016^{33}\end{array}$} \\
\hline & & Moscatilin & & \\
\hline 16 & D.chryseum & Methanol extract & HeLa and U251 cell lines & Pant et al., $2021^{11}$ \\
\hline
\end{tabular}




\begin{tabular}{|c|c|c|c|c|}
\hline \multirow[t]{10}{*}{17} & \multirow[t]{10}{*}{ D. chrysotoxum } & Erianin & \multirow{10}{*}{$\begin{array}{l}\text { T47D, 143B, HUVECs, HeLa, } \\
\text { MG63.2, K562, HL-60, A549, } \\
\text { BEL-7402, SGC-7901 cell lines }\end{array}$} & \multirow{10}{*}{$\begin{array}{l}\text { Chen et al., 2008 } 35 \text {; Hu et al., } \\
2012^{36} \text {; Li et al., 2018 }{ }^{37} \text {; Sun } \\
\text { et al., } 2016^{38} \text {; Wang et al., } \\
2016^{39} \\
\text { Lin et al., } 2005^{27}\end{array}$} \\
\hline & & 1,4,5-trihydroxy-7-methoxy-9H-fluoren-9-one & & \\
\hline & & Dendroflorin & & \\
\hline & & Chrysotoxol B & & \\
\hline & & Confusarin & & \\
\hline & & Moscatin & & \\
\hline & & Epheranthol B & & \\
\hline & & Erianin & & \\
\hline & & Gigantol & & \\
\hline & & Tristin & & \\
\hline 18 & D. denneanum & Polysaccharides & S180 induced mice & Fan \&Luo, $2011^{40}$ \\
\hline 19 & D. densiflorum & Cypripedin & H460 cell line & Wattanathamsan et al., $2018^{41}$ \\
\hline 20 & D. draconis & Gigantol & H460 cell line & $\begin{array}{l}\text { Charoenrungruang et al., } \\
2014 \mathrm{a}^{42}, 2014 \mathrm{~b}^{43} \text {; Unahab- } \\
\text { hokha et al., } 2016^{44}\end{array}$ \\
\hline 21 & D. ellipsophyllum & 4,5,4'-trihydroxy-3,3'-dimethoxybibenzyl & H460, H292, H23 cell lines & $\begin{array}{l}\text { chaotham\&Chanvorchote } \\
2015^{44} \text { : Chaotham et } \\
\text { al.2014 } \\
2018^{46} \text {; Hlosrichok et al., }\end{array}$ \\
\hline 22 & D. falconeri & Dendrofalconerol A & H460 cell line & $\begin{array}{l}\text { Pengpaeng et al., } 2014^{47} \text {, } \\
2015^{48}\end{array}$ \\
\hline \multirow[t]{3}{*}{23} & \multirow[t]{3}{*}{ D. fimbriatum } & $\begin{array}{l}\text { 4-(3-hydroxy-4-methoxyphenethyl)-2,6-dimethox- } \\
\text { ylphenol }\end{array}$ & \multirow[t]{3}{*}{$\begin{array}{l}\text { HL-60, SMMC-7721, A-549, } \\
\text { SW480, MCF-7 cell lines }\end{array}$} & \multirow[t]{3}{*}{ Xu et al., $2014^{49}$} \\
\hline & & Fimbriadimerbibenzyl A, B, E, F and G & & \\
\hline & & Moscatilin & & \\
\hline 24 & D. findlayanum & 4, 4'-dihydroxy-3, 3',5-trimethoxy bibenzyl & $\begin{array}{l}\text { A172, SHSY5Y and HeLa cell } \\
\text { lines }\end{array}$ & Liu et al., $2020^{50}$ \\
\hline 25 & D. formosum & Ethanolic extract & T-cell lymphoma & Prasad \& Koch, $2014^{51}$ \\
\hline \multirow[t]{3}{*}{26} & \multirow[t]{3}{*}{ D. gratiosissimum } & Dengraols A and B & \multirow[t]{3}{*}{ HL-60 } & \multirow[t]{3}{*}{ Zhang et al., $2008^{52}$} \\
\hline & & Gigantol & & \\
\hline & & Moscatilin & & \\
\hline 27 & D. loddigesii & Moscatilin & $\begin{array}{l}\text { HUVECs, A-549, H23, MDA- } \\
\text { MB-231, HCT-116, SCC, A375 } \\
\text { cell lines }\end{array}$ & $\begin{array}{l}\text { Cardile et al., 2020 } \\
\text { et al., } 2013^{54} \text {; Chen et al., } \\
2008^{55} ; \text { Ho \& Chen, } 2003^{56} \text {; } \\
\text { Kowitdamrong et al., } 2013^{57} \text {; } \\
\text { Tsai et al., } 2010^{58}\end{array}$ \\
\hline \multirow[t]{2}{*}{28} & \multirow[t]{2}{*}{ D. longicornu } & Acetone extract & \multirow[t]{2}{*}{ U-251 and HeLa cell lines } & \multirow[t]{2}{*}{ Paudel et al., $2017^{29}$} \\
\hline & & Ethanol extract & & \\
\hline \multirow[t]{4}{*}{29} & \multirow[t]{4}{*}{ D. moniliforme } & Methanol extract & \multirow{4}{*}{$\begin{array}{l}\text { HeLa, HCT-116, MCF-7, U-251, } \\
\text { K562, HSC-T6, BNL CL.2, PC-3 } \\
\text { and DU-145 cell lines, 26-M3.1 } \\
\text { induced mice }\end{array}$} & \multirow{4}{*}{$\begin{array}{l}\text { Hsu et al., 2014 } \\
\text { et al., 2005 }{ }^{60} \text {; Li et al., } \\
2014^{61} ; \text { Paudel et al., } 2018^{62} \text {; } \\
\text { Sánchez-Duffhues et al., } \\
2009^{63} \text {; Sun et al., } 2016^{64} \text {; }\end{array}$} \\
\hline & & Ethanol extract & & \\
\hline & & Denbinobin & & \\
\hline & & Moniliformediquinone & & \\
\hline \multirow[t]{4}{*}{30} & \multirow[t]{4}{*}{ D. nobile } & Denbinobin & \multirow{4}{*}{$\begin{array}{l}\text { K562, PC-3, SNU-484, SK- } \\
\text { Hep-1, HeLa, MCF-7, A549, } \\
\text { HL-60, HepG2 cell lines and } \\
\text { S180 induced mice }\end{array}$} & \multirow{4}{*}{ 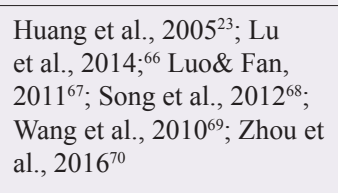 } \\
\hline & & Denobilone A & & \\
\hline & & Lactone derivatives & & \\
\hline & & Polysaccharides & & \\
\hline \multirow[t]{8}{*}{31} & \multirow[t]{8}{*}{ D. officinale } & Aqueous extract & MNNG-induced gastric tumori- & Ren et al., $2020^{71}$; Zhang \\
\hline & & 4,4'-dihydroxy-3,5-dimethoxybibenzyl & $\begin{array}{l}\text { genesis in rats, HeLa cell line, } \\
\text { and SGC-7901 xenograft mice }\end{array}$ & $\begin{array}{l}\text { et al., 2018 } \\
2016^{73} \text {; Zhao et al., }\end{array}$ \\
\hline & & Dendrocandin B & & \\
\hline & & Dendrocandin U & & \\
\hline & & Denofficin & & \\
\hline & & Gigantol & & \\
\hline & & Moscatilin & & \\
\hline & & Polysaccharides & & \\
\hline
\end{tabular}




\begin{tabular}{|c|c|c|c|c|}
\hline 32 & D. polyanthum & Moscatilin & A549, HL-60 cell lines & Hu et al., $2009^{74}$ \\
\hline \multirow[t]{2}{*}{33} & \multirow[t]{2}{*}{ D. pulchellum } & Chrysotoxine & \multirow[t]{2}{*}{ H460, H23 cell lines } & \multirow{2}{*}{$\begin{array}{l}\text { Bhummaphan et al., } \\
2018 ;{ }^{75} \text { Chanvorachote et al., }\end{array}$} \\
\hline & & Moscatilin & & \\
\hline \multirow[t]{6}{*}{34} & \multirow[t]{6}{*}{ D. signatum } & Ethanol extract & \multirow{6}{*}{$\begin{array}{l}\text { MCF-7, NCI-H187, MDA-231, } \\
\text { HepG2 and HT-29 cell lines }\end{array}$} & \multirow{6}{*}{$\begin{array}{l}\text { Chimsook, } 2016^{77} ; \\
\text { Mittraphab et al., } 2016^{78}\end{array}$} \\
\hline & & Dendrosignatol & & \\
\hline & & 3,4-dihydroxy-3',4'-dimethoxybibenzyl & & \\
\hline & & Dendrocandin B & & \\
\hline & & dendrocandin I & & \\
\hline & & Dendrofalconerol A & & \\
\hline \multirow[t]{3}{*}{35} & \multirow[t]{3}{*}{ D. sinense } & 3,4,3'-trimethoxy-5,4'-dihydroxybibenzyl & \multirow{3}{*}{$\begin{array}{l}\text { SGC-7901, BEL-7402, K562 } \\
\text { cell lines }\end{array}$} & \multirow[t]{3}{*}{ Chen et al., $2014^{79}$} \\
\hline & & 5,3'-dihydroxy-3,4-dimethoxy-bibenzyl & & \\
\hline & & Longicornuol A & & \\
\hline 36 & D. transparens & Methanol extract & HeLa and U251 cell lines & Joshi et al., $2020^{9}$ \\
\hline \multirow[t]{3}{*}{37} & \multirow{3}{*}{$\begin{array}{l}\text { Ephemerantha } \\
\text { lonchophylla }\end{array}$} & Ephemeranthone & \multirow[t]{3}{*}{ K562 cell line } & \multirow[t]{3}{*}{ Chen et al., $2000^{80}$} \\
\hline & & Erianthridin & & \\
\hline & & Denbinobin & & \\
\hline 38 & Eulophia macrobulbon & Ethanol extract and fractions & $\begin{array}{l}\text { MCF- } 7, \mathrm{HeLa} \text { and } \mathrm{CaCo}-2 \text { cell } \\
\text { lines }\end{array}$ & Schuster et al., $2017^{81}$ \\
\hline \multirow[t]{2}{*}{39} & \multirow[t]{2}{*}{ E. nuda } & 9,10-dihydro-2,5-dimethoxyphenanthrene-1,7-diol & \multirow{2}{*}{$\begin{array}{l}\text { MCF- } 7 \text { and MDA-MB-231 cell } \\
\text { lines }\end{array}$} & \multirow{2}{*}{$\begin{array}{l}\text { Shriram et al., } 2010^{82} \text {; Bhatt } \\
\text { et al., } 2018^{83}\end{array}$} \\
\hline & & Alcohol extact & & \\
\hline 40 & Gastrodiaelata & N6-(4-hydroxybenzyl) adenine riboside & PC12 cell line & Huang et al., $2004^{21}$ \\
\hline \multirow[t]{4}{*}{41} & \multirow[t]{4}{*}{ Gymnadeniaconopsea } & Gymconopin A & & \multirow[t]{4}{*}{ Matsuda et al., $2004^{84}$} \\
\hline & & Gymconopin B & & \\
\hline & & Gymconopin D & & \\
\hline & & $\begin{array}{l}\text { Dihydroxy-2,6-bis(4-hydroxybenzyl)-5-methoxy- } \\
\text { bibenzyl }\end{array}$ & & \\
\hline \multirow[t]{6}{*}{42} & \multirow[t]{6}{*}{ Maxillaria densa } & 2,5-Dihydroxy-3, 4-Dimethoxyphenanthrene & & \multirow[t]{6}{*}{ Decigsa-campos et al., $2007^{85}$} \\
\hline & & $\begin{array}{l}\text { 9,10-dihydro-2,5-dihydroxy-3, } \\
\text { 4-dimethoxyphenanthrene }\end{array}$ & & \\
\hline & & Nudol & & \\
\hline & & Gymnopusin & & \\
\hline & & Erianthridin & & \\
\hline & & Fimbriol A & & \\
\hline 43 & Papilionanthe uniflora & Methanol extract & HeLa cell line & Joshi et al., $2020^{9}$ \\
\hline 44 & Pholidota articulata & Methanol extract & HeLa cell line & Joshi et al., $2020^{9}$ \\
\hline \multirow[t]{4}{*}{45} & P. yunnanensis & 2,4,7-Trihydroxy-9,10-Dihydrophenanthrene & & Guo et al., $2007^{86}$ \\
\hline & & 3,7-dihydroxy-2,4,8-Trimethoxyphenanthrene & & \\
\hline & & Coelonin & & \\
\hline & & 3,7-Dihydroxy-2,4-dimethoxyphenanthrene & & \\
\hline 46 & Rhyncostylish retusa & Methanol extract & & Radhika et al., $2013^{87}$ \\
\hline 47 & Spiranthes australis & $\begin{array}{l}\text { (2S)-5,2',6'-trihydroxy-6-lavandulyl-4"-( } \gamma, \gamma \\
\text {-dimethylallyl)-2",2"-dimethylpyrano-[5",6": 7,8]- } \\
\text { flavanone }\end{array}$ & $\begin{array}{l}\text { A549, BEL-7402, SGC-7901, } \\
\text { MCF-7, HT-29, K562, and A498 } \\
\text { cell lines }\end{array}$ & Peng et al., $2007^{23}$ \\
\hline 48 & Vanda cristata & Methanol extract & HeLa and U251 cell lines & Joshi et al., 2020 \\
\hline
\end{tabular}




\section{CONCLUSION}

It is documented that orchids are rich in potential anticancer agents and are on the forefront in terms of offering possibilitiesfornewanticancerdrugs. Manyexisting anticancer remedies based on traditional medicine are of orchid origin. In fact, orchids have exhibited anticancer activities in animal models of leukemia, skin cancer and sarcomas. For those plants which have shown promising results, further investigation has ensued but deeper investigations for single active molecular moiety must be undertaken. While cancer exerts a high mortality rate and may not be treatable by the usual arsenal of an oncologist, it is likely that orchids and their components may play a role in palliative care or reduce the side effects associated with currently available cancer treatments. By generating awareness regarding the use of herbs and exploring their natural product properties, healthcare professionals can play a significant critical role as knowledge resource for the masses. From literature-based information, our research findings show that particular orchids, used since ancient times in traditional medicine under various formulations, contain potential anticancer drug candidates in their plant material and may also experience eventual utilization as herbal product, depending on the specific data collected.

\section{ACKNOWLEDGEMENT}

The authors gratefully acknowledge Dr. Sven H Wagner for the constructive feedback and suggestions on the manuscript.

\section{REFERENCES}

1. Bray F, Ferlay J, Soerjomataram I, Siegel RL, Torre LA, Jemal A.Global cancer statistics 2018: GLOBOCAN estimates of incidence and mortality worldwide for 36 cancers in 185 countries.CA: a cancer journal for clinicians.2018;68(6), 394-424.

2. Siegel RL, Miller KD, Goding SA, Fedewa SA, Butterly LF, Anderson JC, Jemal A. Colorectal cancer statistics, 2020. CA: a cancer journal for clinicians.2020; 70(3), 145-164.

3. Guilbaud N, Kraus BL, Meyer LF, Malivet V, Chacun C, Jan M,Atassi G. Marked antitumor activity of a new potent acronycine derivative in orthotopic models of human solid tumors. Clinical Cancer Research,2011;7(8), 2573-2580.

4. Moraes DFC,Mesquita LSS,Amaral FM,Malik S. Anticancer drugs from plants. In Biotechnology and Production of Anti-Cancer Compounds.2017;(pp. 121-142).Springer, Cham.
5. Greenwell M, Rahman PK.Medicinal plants: their use in anticancer treatment. International Journal of Pharmaceutical Sciences and Research.2015; 6(10), 4103.

6. Vital PG, Rivera, WL. Antimicrobial activity and cytotoxicity of Chromolaena odorata (L. f.) King and Robinson and Uncaria perrottetii (A. Rich) Merr. Extracts. Journal of medicinal plants Research.2009; 3(7), 511-518

7. Gordon MC, David J.Plants as a source of anti-cancer agents.Journal of Ethnopharmacology.2005; 100, 72.

8. Paudel MR, Bhattarai HD, Pant B. Traditionally used medicinal Dendrobium: a promising source of active anticancer constituents. Orchids Phytochemistry, Biology and Horticulture: Fundamentals and Applications.2020; 1-26.

9. JoshiPR, PaudelMR, Chand MB, Pradhan S, Pant KK, Joshi GP, Pant B. Cytotoxic effect of selected wild orchids on two different human cancer cell lines. 2020; 6(5), e03991.

10. Thapa, BB, Thakuri LS, Joshi PR, Chand K, Rajbahak S, Sah AK, Pant B. Ex-situ conservation and cytotoxic activity assessment of native medicinal orchid: Coelogyne stricta. Journal of Plant Biotechnology.2020; 47(4), 330-336

11. Pant B, Joshi PR, Maharjan S, Thakuri LS, Pradhan S, Shah S,Pant B. Comparative Cytotoxic Activity of Wild Harvested Stems and In Vitro-Raised Protocorms of Dendrobium chryseum Rolfe in Human Cervical Carcinoma and Glioblastoma Cell Lines. Advances in Pharmacological and Pharmaceutical Sciences, 2021.

12. Chaotham C, Chanvorachote P. A bibenzyl from Dendrobium ellipsophyllum inhibits migration in lung cancer cells. Journal of Natural Medicines.2015; 69, 565574.

13. Chen YG, $X u J J, Y u ~ H$, Qing C, Zhang YL, Liu Y, Wang JH. 3, 7-Dihydroxy-2, 4, 6-trimethoxyphenanthrene, a new phenanthrene from Bulbophyllum odoratissimum. Journal of the Korean Chemical Society.2007;51(4), 352-355.

14. Morita H, Koyama K, Sugimoto Y, Kobayashi Jl. Antimitotic activity and reversal of breast cancer resistance proteinmediated drug resistance bystilbenoids from Bletilla striata. Bioorganic \& Medicinal Chemistry Letters.2005;15(4), 1051-1054

15. Feng GS, Li X, Zheng CS, Zhou CK, Liu X,Wu HP. Mechanism of inhibition of tumor angiogenesis by Bletilla colloid: an experimental study. 2003; 83(5), 412-416.

16. Xue Z, Li S, Wang S, Wang Y, Yang Y, Shi J, He L.Mono-, bi-, and triphenanthrenes from the tubers of Cremastra appendiculata. Journal of Natural Products.2006;69(6), 907-913 
17. Lee YH, Park JD, Beak NI, Kim SI, Ahn BZ . In vitro and in vivo antitumoral phenanthrenes from the aerial parts of Dendrobium nobile. Planta Medica.1995; 61(02), 178180.

18. Li Y, Wang H, \& Liu G. Erianin induces apoptosis in human leukemia HL-60 cells. Acta Pharmacologica Sinica.2001; 22(11), 1018-1022.

19. Gong YQ, Fan $Y$, Wu DZ, Yang H, Hu ZB, Wang ZT. In vivo and in vitro evaluation of erianin, a novel antiangiogenic agent. European Journal of Cancer.2004.

20. Yang L, Qin LH, Bligh SA, Bashall A, Zhang CF, Zhang M, $\& \mathrm{Xu}$ LS. A new phenanthrene with a spirolactone from Dendrobium chrysanthum and its antiinflammatory activities.Bioorganic \& Medicinal Chemistry Letters.2006; 14(10), 3496-3501

21. Huang NK, Lin YL, Cheng JJ, Lai WL.Gastrodia elata prevents rat pheochromocytoma cells from serumdeprived apoptosis: the role of the MAPK family. Life Sciences.2004; 75(13), 1649-1657

22. Heo JC, Woo SU, Son M, Park JY, Choi WS, Chang KT, Lee, $\mathrm{SH}$. Anti-tumor activity of Gastrodia elata Blume is closely associated with a GTP-Ras dependent pathway.Oncology Reports.2007; 8(4), 849-853.

23. Peng J, Xu Q, Xu Y, Qi Y, Han X, Xu L.A new anticancer dihydroflavanoid from the root of Spiranthes australis (R. Brown) Lindl.Natural Product Research.2007;21(7), 641645.

24. Majumder PL, Sen S, Majumder S. Phenanthrene derivatives from the orchid Coelogyne cristata. Phytochemistry.2007;58(4), 581-586.

25. Majumder PL, Majumder S, Sen S. Triterpenoids from the orchids Agrostophyllum brevipes and Agrostophyllum callosum.Phytochemistry.2003;62(4), 591-596.

26. Tseng CC, Shang HF, Wang LF, Su B, Hsu CC, Kao HY, Cheng KT. Antitumor and immunostimulating effects of Anoectochilus formosanus Hayata.Phytomedicine.2006;13(5), 366-370.

27. Lin YL, Chen WP, Macabalang AD. Dihydrophenanthrenes from Bletilla formosana. Chemical and pharmaceutical bulletin.2005; 53(9), 1111-1113.

28. Wu B, He S, Pan YJ. New dihydrodibenzoxepins from Bulbophyllum kwangtungense. PlantaMedica.2006;72(13), 1244-1247.

29. Paudel MR, Chand MB, Pant B, Pant B. Cytotoxic activity of antioxidant-rich Dendrobium longicornu. Pharmacognosy Journal.2007;9, 499-503.

30. Klongkumnuankarn P, Busaranon K, Chanvorachote
P, Sritularak B, Jongbunprasert V, Likhitwitayawuid $\mathrm{K}$. Cytotoxic and antimigratory activities of phenolic compounds from Dendrobium brymerianum.EvidenceBased Complementary and Alternative Medicine. 2015;350-410.

31. Zheng Q, Qiu D, Liu X, Zhang L, Cai S, Zhang X. Antiproliferative effect of Dendrobium catenatum Lindley polypeptides against human liver, gastric and breast cancer cell lines. Food \& function,2015; 6(5), 1489-1495.

32. Paudel MR, Chand MB, Pant B,Pant B. Assessment of antioxidant and cytotoxic activities of extracts of Dendrobium crepidatum. Biomolecules.2019;9, 478.

33. Prasad R,Koch B. In vitro anticancer activities of ethanolic extracts of Dendrobium crepidatum and Dendrobium chrysanthum against T-cell lymphoma.Journal of Cytology \& Histology.2016;7,432.

34. Nam B, Ryu SM, Lee D, Jung $\mathrm{CH}$, Jin $\mathrm{CH}$, Kim JB, Han AR. Identification of two new phenanthrenes from Dendrobii Herba and their cytotoxicity towards human hypopharynx squamouscarcinomacell(FaDu).Molecules.2019;24,2339

35. Chen TH, PanSL, Guh JH, Liao CH, Huang DY, Chen CC, Teng CM.Moscatilin inducesapoptosisinhumancolorectalcancer cells: a crucial role of c-Jun $\mathrm{NH}$ 2-terminal protein kinase activation caused by tubulin depolymerization and DNA damage. Clinical Cancer Research.2008;14, 4250-4258.

36. Hu J, Fan W, Dong F, Miao Z, Zhou J. Chemical Components of Dendrobium chrysotoxum. Chinese Journal of Chemistry.2012; 30, 1327-1330.

37. Li M, He Y, Peng C, Xie X, Hu G. Erianin inhibits human cervical cancer cell through regulation of tumor protein p53 via the extracellular signal regulated kinase signaling pathway.Oncology Letters.2018;16, 5006-5012.

38. Sun J, Guo Y, Fu X, Wang Y, Liu Y, Huo B, Hu X.Dendrobium candidum inhibits MCF-7 cells proliferation by inducing cell cycle arrest at $\mathrm{G} 2 / \mathrm{M}$ phase and regulating key biomarkers. OncoTargets and Therapy.2016;9, 21-30.

39. Wang $H$, Zhang $T$, Sun W, Wang $Z$, Zuo D, Zhou $Z$, Hua Y. Erianin induces G2/M-phase arrest, apoptosis, and autophagy via the ROS/JNK signaling pathway in human osteosarcoma cells in vitro and in vivo. Cell Death and Disease.2016; 7, e2247.

40. Fan Y, LuoA. Evaluation ofanti-tumoractivityofwater-soluble polysaccharides from Dendrobium denneanum. African Journal of Pharmacy and Pharmacology.2011;5, 415-420.

41. Wattanathamsan O, Treesuwan S, Sritularak B, Pongrakhananon V.Cypripedin, a phenanthrenequinone from Dendrobium densiflorum, sensitizes non-small cell lung cancer $\mathrm{H} 460$ cells to cisplatin-mediated apoptosis. 
Journal of Natural Medicines,2018.

42. Charoenrungruang $S$, Chanvorachote $P$, Sritularak $B$, Pongrakhananon V. Gigantol-induced apoptosis in lung cancer cell through mitochondrial-dependent pathway. Thai Journal of Pharmaceutical Sciences 2014;38, 67-73.

43. FanY,LuoA. Evaluation ofanti-tumoractivityofwater-soluble polysaccharides from Dendrobium denneanum. African Journal of Pharmacy and Pharmacology.2011;5, 415-420.

44. Unahabhokha T, Chanvorachote P,Pongrakhananon V. The attenuation of epithelial to mesenchymal transition and induction of anoikis by gigantol in human lung cancer H460 cells. Tumor Biology.2016; 37, 8633-8641.

45. Charoenrungruang $S$, Chanvorachote $P$, Sritularak $B$, Pongrakhananon $\mathrm{V}$.Gigantol-induced apoptosis in lung cancer cell through mitochondrial-dependent pathway. Thai Journal of Pharmaceutical Sciences,.2014;38, 67-73.

46. Unahabhokha T, Chanvorachote P, Pongrakhananon V. The attenuation of epithelial to mesenchymal transition and induction of anoikis by gigantol in human lung cancer H460 cells. Tumor Biology.2016;37, 8633-8641.

47. Pengpaeng $P, \quad$ Sritularak B, Chanvorachote P. Dendrofalconerol A sensitizes anoikis and inhibits migration in lung cancer cells. Journal of Natural Medicines, 2014;69, 178-190.

48. Pengpaeng $P$, Sritularak B,Chanvorachote $P$. Dendrofalconerol: A suppresses migrating cancer cells via EMT and integrin proteins. Anticancer Research.2015;35, 201-206.

49. Xu FQ, Xu, FC, Hou B, Fan WW, Zi CT, Li Y, Zuo ZL. Cytotoxic bibenzyl dimers from the stems of Dendrobium fimbriatum Hook. Bioorganic \& Medicinal Chemistry Letters, 2014;24, 5268-5273.

50. Pengpaeng, P, Sritularak B, Chanvorachote P. Dendrofalconerol A sensitizes anoikis and inhibits migration in lung cancer cells. Journal of Natural Medicines, 2014;69, 178-190.

51. Pengpaeng $P, \quad$ Sritularak B, Chanvorachote P. Dendrofalconerol A suppresses migrating cancer cells via EMT and integrin proteins. Anticancer Research,2015; 35, 201-206.

52. Zhang $C F$, Wang $M$, Wang $L$, Linuma $M$, Zhang, $M, X u$ LS, Wang ZT .Chemical constituents of Dendrobium gratiosissimum and their cytotoxic activities. Indian Journal of Chemistry,2008; 47, 952-956.

53. Cardile V, Avola, Graziano AC, Russo A. Moscatilin, a bibenzyl derivative from the orchid Dendrobium loddigesii, induces apoptosis in melanoma cells. Chemico-Biological Interactions.2020;323, 109075.

54. Charoenrungruang $S$, Chanvorachote $P$, Sritularak B, Pongrakhananon V. Gigantol, a bibenzyl from Dendrobium draconis, inhibits the migratory behavior of non-small cell lung cancer cells. Journal of Natural Products.2014;77, 1359-1366

55. Prasad R, Koch B. Antitumor activity of ethanolic extract of Dendrobium formosum in T-celllymphoma: An invitroand in vivo study. BioMed research international. 2014; 753451.

56. Ho CK, Chen CC. Moscatilin from the orchid Dendrobrium loddigesii is a potential anticancer agent. Cancer Investigation.2003; 21, 729-736.

57. Chaotham C, Chanvorachote P.A bibenzyl from Dendrobium ellipsophyllum inhibits migration in lung cancer cells Journal of Natural Medicines.2015; 69, 565-574.

58. Chen CA, Chen CC, Shen CC, Chang HH, Chen YJ. Moscatilin induces apoptosis and mitotic catastrophe in human esophageal cancer cells. 2010;Journal of Medicinal Food.2013;16, 869-877.

59. Chen Y, Li Y, Qing C, Zhang Y, Wang L, Liu Y. 1,4,5-Trihydroxy7-methoxy-9H-fluoren-9-one, a new cytotoxic compound from Dendrobium chrysotoxum.Food Chemistry.2008;108, 973-976

60. Tsai AC, Pan SL, Liao CH, Guh JH, Wang SW, Sun HL, Chang, YL. Moscatilin a bibenzyl derivative from the India orchid Dendrobrium loddigesii, suppresses tumor angiogenesis and growth in vitro and in vivo. Cancer Letters. 292, 163170.

61. Kowitdamrong A, Chanvorachote $P$, Sritularak B, Pongrakhananon V. Moscatilin inhibits lung cancer cell motility and invasion via suppression of endogenous reactive oxygen species.2013;BioMed Research International, 2013, 765894.

62. Paudel MR, Chand MB, Pant B, Pant B. Antioxidant and cytotoxic activities of Dendrobium moniliforme extracts and the detection of related compounds by GC-MS. BMC Complementary and Alternative Medicine.2018;134.

63. Hsu JL, Lee YJ, Leu WJ, Dong Y, Pan SL, Uang BJ, Guh $\mathrm{JH}$. Moniliformediquinone induces in vitro and in vivo antitumor activity through glutathione involved DNA damage response and mitochondrial stress in human hormone refractory prostate cancer.2014; The Journal of Urology, 191, 1429-1438.

64. Zhao X, Sun P, Qian Y, Suo H. D. Candidum has in vitro anticancer effects in HCT-116 cancer cells and exerts in vivo anti-metastatic effects in mice.2014; Nutrition Research and Practice, 8(5), 487. 
65. Li G, Sun P, Zhou Y, Zhao X, Chen F. Preventive effects of Dendrobium candidum Wall ex Lindl.on the formation of lung metastases in BALB/c mice injected with 26-M3.1 colon carcinoma cells.2014;Oncology Letters, 8, 18791885.

66. SánchezDuffhues $G$, Calzado MA, De Vinuesa AG, Appendino G, Fiebich BL, Loock U, Muñoz E. Denbinobin inhibits nuclear factor-KB and induces apoptosis via reactive oxygen species generation in human leukemic cells.2009;Biochemical Pharmacology, 77, 1401-1409.

67. Sun J, Fu X, Wang Y, Liu Y, Zhang Y, Hao T, Hu X. Erianin inhibits the proliferation of T47D cells by inhibiting cell cycles, inducing apoptosis and suppressing migration. American Journal of Translational Research.2016; 8, 30773086.

68. Wang JH, Luo JP, Zha XQ, Feng BJ. Comparison of antitumor activities of different polysaccharide fractions from the stems of Dendrobium nobile Lindl. Carbohydrate Polymer.2010; 79, 114-118. 64.

69. Zhou XM, Zheng CJ, Wu JT, Chen GY, Chen J, Sun CG. Five new lactone derivatives from the stems of Dendrobium nobile. Fitoterapia.2016;115, 96-100.

70. Ren G, Deng WZ, Xie YF, Wu CH, Li WY, Xiao CY,Chen YL. Bibenzyl derivatives from leaves of Dendrobium officinale. Natural Product Communications.2020;15, 1-5.

71. Zhang L, Wang F, Ren X. Inhibitory effect of Dendrobium officinale polysaccharide on human gastric cancer cell xenografts in nude mice.Food Science and Technology.2018;38, 78-83.

72. Zhao Y, Liu Y, Lan XM, Xu GL, Sun YZ, Li F, Liu HN. Effect of Dendrobium officinale extraction on gastric carcinogenesis in rats. Evidence-Based Complementary and Alternative Medicine.2016;

73. Hu JM, Zhao YX, Miao ZH, Zhou J. Chemical components of Dendrobium polyanthum.Bulletin of the Korean Chemical Society.2009; 30, 2098-2100.

74. Zhang L, Wang F, Ren X.Inhibitory effect of Dendrobium officinale polysaccharide on human gastric cancer cell xenografts in nude mice.Food Science and Technology.2018;38, 78-83.

75. Zhao Y, Liu Y, Lan XM, Xu GL, Sun YZ, Li F, Liu HN. Effect of Dendrobium officinale extraction on gastric carcinogenesis in rats. Evidence-Based Complementary and Alternative Medicine. 2016.

76. Luo A,Fan Y. Immune stimulating activity of water-soluble polysaccharide fractions from Dendrobium nobile Lindl. African Journal of Pharmacy and Pharmacology.2011; 5-625-631.
77. Hsu JL, Lee YJ, Leu WJ, Dong YS, Pan SL, Uang BJ, Guh $\mathrm{JH}$. Moniliformediquinone induces in vitro and in vivo antitumor activity through glutathione involved DNA damage response and mitochondrial stress in human hormone refractory prostate cancer.2014; The Journal of Urology, 191, 1429-1438.

78. Ren G, Deng WZ, Xie YF, Wu CH, Li WY, Xiao CY, Chen YL. Bibenzyl derivatives from leaves of Dendrobium officinale. Natural Product Communications.2020;15, 1-5.

79. Chen XJ, Mei WL, Cai CH, Guo ZK, Song XQ, Dai HF. Four new bibenzyl derivatives from Dendrobium sinense. Phytochemistry Letter.2014;9, 107-112.

80. Cardile V, Avola R, Graziano AC, Russo A .Moscatilin, a bibenzyl derivative from the orchid Dendrobium loddigesii, induces apoptosis in melanoma cells. Chemico-Biological Interactions.2020;323, 109075.

81. Schuster R, Zeindl L, Holzer W, Khumpirapang N, Okonogi $\mathrm{S}$, Viernstein H, Mueller M. Eulophia macrobulbon-an orchid with significant anti-inflammatory and antioxidant effect and anticancerogenic potential exerted by its root extract. Phytomedicine.2017;24, 157-165.

82. Shriram V, Kumar V, Kishor PK, Suryawanshi SB, Upadhyay AK, Bhat MK. Cytotoxic activity of 9, 10-dihydro-2, 5-dimethoxyphenanthrene-1, 7-diol from Eulophia nuda against human cancer cells.Journal of ethnopharmacology. 128(1),2010; 251-253.

83. Bhatt, D. R., Jethva, K. D., \&Zaveri, M. N. (2018). In-vitro cytotoxicity studies of the therapeutic orchid: Eulophia nuda. Journal of Pharmacognosy and Phytochemistry.2018; 7(4), 680-683.

84. Matsuda H, Morikawa T, Xie H, Yoshikawa M. Antiallergic phenanthrenes and stilbenes from the tubers of Gymnadenia conopsea.2004; Planta medica, 70(09), 847855.

85. Déciga-Campos $M$, Palacios-Espinosa JF, Reyes-Ramírez A, Mata R. Antinociceptive and anti-inflammatory effects of compounds isolated from Scaphyglottis livida and Maxillaria densa. Journal of Ethnopharmacology.2007; 114(2), 161-168.

86. Guo XY, Wang J, Wang NL, Kitanaka S, Yao XS. 9,10Dihydrophenanthrene derivatives from Pholidota yunnanensis and scavenging activity on DPPH free radical. Journal of Asian Natural Products Research.2007; 9(2), 165-174.

87. Radhika B, Murthy J, Grace DN. Antifungal and cytotoxic activities of medicinal important orchid Rhynchostylis retusa Blume.Internal Journal of Advanced Research.2013; 1, 31-35. 